TITLE:

\title{
Interface and vortex motion in the two-component complex dissipative Ginzburg-Landau equation in two-dimensional space
}

$\operatorname{AUTHOR(S):~}$

Yabunaka, Shunsuke

\section{CITATION:}

Yabunaka, Shunsuke. Interface and vortex motion in the two-component complex

dissipative Ginzburg-Landau equation in two-dimensional space. Physical Review E 2014, 90(4): 042925.

ISSUE DATE:

2014-10-28

URL:

http://hdl.handle.net/2433/191211

RIGHT:

(C)2014 American Physical Society 


\title{
Interface and vortex motion in the two-component complex dissipative Ginzburg-Landau equation in two-dimensional space
}

\author{
Shunsuke Yabunaka \\ Yukawa Institute for Theoretical Physics, The Kyoto University, Kitashirakawa Oiwake-Cho, 606-8502 Kyoto, Japan
}

(Received 14 April 2014; revised manuscript received 6 July 2014; published 28 October 2014)

\begin{abstract}
We study interface and vortex motion in the two-component dissipative Ginzburg-Landau equation in twodimensional space. We consider cases where the whole system is divided into several domains, and we assume that these domains are separated by interfaces and each domain contains quantized vortices. The equations for interface and vortex motion will be derived by means of a variational approach by Kawasaki. These equations indicate that, along an interface, the phase gradient fields of the complex order parameters is parallel to the interface. They also indicate that the interface motion is driven by the curvature and the phase gradient fields along the interface, and vortex motion is driven by the phase gradient field around the vortex. With respect to the static interactions between defects, we find an analogy between quantized vortices in a domain and electric charges in a vacuum domain surrounded by a metallic object in electrostatic. This analogy indicates that there is an attractive interaction between an interface and a quantized vortex with any charge. We also discuss several examples of interface and vortex motion.
\end{abstract}

DOI: 10.1103/PhysRevE.90.042925

PACS number(s): $89.75 . \mathrm{Kd}$

\section{INTRODUCTION}

Motion of topological defects has been studied extensively. Its understanding is crucial to theoretical description of ordering process in phase transitions and pattern formations in nonequilibrium open systems. As an earlier work, Kawasaki and Ohta derived the equations of interface motion by reducing the freedoms of the so-called Model $\mathrm{H}$, which describes phase-separating fluid systems [1]. Motion of a quantized vortex in the complex Ginzburg-Landau model has been studied by several authors $[2,3]$. Onuki derived the equation of vortex motion in the so-called Model F, which describes the superfluid transition of ${ }^{4} \mathrm{He}$ near the $\lambda$ point, to discuss the mutual friction between the normal component and the superfluid component [2]. Motion of disclinations in liquid crystals was also studied by many authors [4-6]. Finally, we note that Kawasaki formulated a theory that describes motion of general kinds of topological defects by a variational approach considering the dissipation of the free energy due to the motion of defects [7]. This method enables us to describe motion of topological defects under general configurations in a simple way.

Different kinds of topological defects can coexist in many systems. For example, in incommensurate modulated systems [8], mixtures of a liquid crystal and an isotropic fluid $[9,10]$, and phase separating two-component BoseEinstein condensates [11], interfaces, and line defects coexist generally in three-dimensional space. It is of great interest how different kinds of defects interact with each other. However, there have been few theoretical works on this subject. Numerical simulations of phase transition dynamics in mixtures of a liquid crystal and an isotropic fluid were carried out and coexistence of interfaces and disclinations was observed $[9,10]$. Dynamics in the presence of interplays between interfaces and vortices in the multicomponent Bose-Einstein condensates was studied by several authors. Vortex motion in the Bose-Einstein condensation in the presence of a fixed plane boundary was also examined [12]. Formation of quantized vortices due to Rayleigh-Taylor instability of the interface between condensates of different kinds of components was also investigated numerically [11]. However, the equations of interface and vortex motion in systems where interfaces can freely deform have not been derived analytically so far.

The purpose of this paper is to investigate interface and vortex motion in the two-component complex Ginzburg-Landau equation with dissipation, because the system is one of the simplest systems where different kinds of topological defects coexist and it is also related to dissipative dynamics of phase separating two component Bose-Einstein condensations. We will derive the equations of motion of vortices and interfaces for general configurations following Kawasaki's variational method [7] mentioned above. These equations indicate that, along an interface, the phase gradient fields of the complex order parameters is parallel to the interface. They also indicate that the interface motion is driven by the energy density difference between the two components, the curvature and the phase gradient fields along the interface, and the motion of a vortex in a domain is driven by the phase gradient field around the vortex. With respect to the static interactions between defects, we find an analogy between quantized vortices in a domain and electric charges in a vacuum domain surrounded by a metallic object in electrostatic. This analogy indicates that there is an attractive interaction between an interface and a quantized vortex with any charge.

\section{MODEL}

We employ the following free-energy functional for twocomponent complex fields $\psi_{i}(i=1,2)$ in two-dimensional space:

$$
\begin{aligned}
H= & \int d \vec{r}\left[\sum_{i=1,2} \frac{a_{i}}{2}\left|\psi_{i}\right|^{2}+\sum_{i, j=1,2} \frac{g_{i, j}}{4}\left|\psi_{i}\right|^{2}\left|\psi_{j}\right|^{2}\right. \\
& \left.+\sum_{i=1,2} \frac{1}{2} K_{i}\left|\vec{\nabla} \psi_{i}\right|^{2}\right] .
\end{aligned}
$$


Here we assume $a_{i}<0, g_{i, j}>0, g_{1,1} g_{2,2}<g_{1,2}^{2}$, and $K_{i}>0$ for $i, j=1,2$. Under this condition, the free energy minima are taken at spatially uniform configurations $\psi_{i}(\vec{r})=$ $\left(\psi_{1}^{\min }, \psi_{2}^{\min }\right)$ with

$$
\left(\left|\psi_{1}^{\min }\right|,\left|\psi_{2}^{\min }\right|\right)=\left(\sqrt{\frac{\left|a_{1}\right|}{g_{1,1}}}, 0\right) \equiv\left(M_{1}, 0\right),
$$

or

$$
\left(\left|\psi_{1}^{\min }\right|,\left|\psi_{2}^{\min }\right|\right)=\left(0, \sqrt{\frac{\left|a_{2}\right|}{g_{2,2}}}\right) \equiv\left(0, M_{2}\right),
$$

which means that the two components are phase separated [13]. We will confirm that these single-component solutions are more stable than the two-component solutions that satisfy both $\left|\psi_{1}^{\min }\right| \neq 0$ and $\left|\psi_{2}^{\min }\right| \neq 0$ in Appendix A.

We give time evolution equations for the complex fields $\psi_{1}$ and $\psi_{2}$ as

$$
\begin{gathered}
\frac{\partial \operatorname{Re} \psi_{i}}{\partial t}=-\operatorname{Re} L_{i} \frac{\delta H}{\delta \operatorname{Re} \psi_{i}}+\operatorname{Im} L_{i} \frac{\delta H}{\delta \operatorname{Im} \psi_{i}}, \\
\frac{\partial \operatorname{Im} \psi_{i}}{\partial t}=-\operatorname{Re} L_{i} \frac{\delta H}{\delta \operatorname{Im} \psi_{i}}-\operatorname{Im} L_{i} \frac{\delta H}{\delta \operatorname{Re} \psi_{i}},
\end{gathered}
$$

where $i=1,2$. We assume that $\operatorname{Re} L_{i}>0$ for $i=1,2$ so that the free energy decreases in the time evolution. The above equations with $\operatorname{Re} L_{i}=0$ reduce to the two-component nondissipative Gross-Pitaevskii equations for macroscopic wave functions $\psi_{i}(i=1,2)$ without an external potential. The dissipative coefficients $\operatorname{Re} L_{i}>0$ are added if one needs to describe the dissipation of superfluid components phenomenologically. We can rewrite the above equations more compactly as

$$
\frac{\partial \psi_{i}}{\partial t}=-L_{i} \frac{\delta H}{\delta \psi_{i}^{*}} .
$$

Here we have defined the derivative $\delta H / \delta \psi_{i}^{*}$ and $\delta H / \delta \psi_{i}$ as

$$
\begin{aligned}
\frac{\delta H}{\delta \psi_{i}^{*}} & =\frac{\delta H}{\delta \operatorname{Re} \psi_{i}}+i \frac{\delta H}{\delta \operatorname{Im} \psi_{i}}, \\
\frac{\delta H}{\delta \psi_{i}} & =\frac{\delta H}{\delta \operatorname{Re} \psi_{i}}-i \frac{\delta H}{\delta \operatorname{Im} \psi_{i}} .
\end{aligned}
$$

In this paper, we consider cases where the whole system is divided into several domains, and we assume that these domains are separated by interfaces and each domain contains quantized vortices. In two-dimensional space, an interface is a line defect, and a quantized vortex is a point defect. Except near the interfaces and the quantized vortices, the amplitudes of the complex fields in the first-component domains and the two-component domains are given by $\left(\left|\psi_{1}\right|,\left|\psi_{2}\right|\right)=\left(M_{1}, 0\right)$ or $\left(\left|\psi_{1}\right|,\left|\psi_{2}\right|\right)=\left(0, M_{2}\right)$.

\section{DERIVATION OF THE EQUATIONS OF INTERFACE AND VORTEX MOTION}

In this section we will derive the equations of interface and vortex motion. We assume that the time scale of defect motion is so large that it is well separated from time scales of the other excitation modes. This assumption can be justified when the curvature radius of interfaces and distance between topological defects are much larger than the thickness of interface $\xi_{i} \equiv$ $\sqrt{K_{i} /\left|a_{i}\right|}$, which is of the same order as the radius of vortex cores. Due to this assumption, we are able to ignore all the other excitations than defect motion such as spin waves and modulation of amplitude because they decay much faster than defect motion. In such cases, we can describe dynamics of the system in terms of defect motion as we will do in Eq. (14) below.

First, we will derive the equation of motion for a point vortex in the $i$ th component domain $D$ by following Kawasaki's variational method [7]. Inside the domain, $\left|\psi_{i}\right| \cong M_{i}$ and $\left|\psi_{i^{\prime}}\right| \cong 0$ for $i^{\prime} \neq i$. We assume that there are $N_{D}$ vortices inside the domain $D$. We consider only vortices with the topological charge $n= \pm 1$ because a vortex with $|n|>1$ is energetically unstable and spontaneously splits into $|n|$ vortices with the topological charge $n /|n|$. We denote the positions and the charge of the $j$ th vortex inside the domain $D$ as

$$
\begin{gathered}
n_{j}= \pm 1, \\
\vec{r}_{j}=\left(x_{j}, y_{j}\right) .
\end{gathered}
$$

Inside the domain $D$, the deviation of the $i$ th component order parameter due to the virtual displacement $\delta \eta_{\alpha}$ of the vortices are given by

$$
\begin{gathered}
\delta \psi_{i}(\vec{r})=\sum_{\alpha=x, y} \sum_{j=1}^{N_{D}} \gamma_{i \alpha}(\vec{r}, j) \delta \eta_{\alpha}(j), \\
\delta \psi_{i^{\prime}}(\vec{r}) \cong 0,
\end{gathered}
$$

where $i^{\prime} \neq i$ and $\gamma_{i \alpha}(\vec{r}, j)$ represents the Goldstone mode due to the displacement of the $j$ th vortex. The free-energy deviation due to this displacement of the vortices inside the domain $D$ is given as

$$
\begin{aligned}
\delta H & =\sum_{i^{\prime}=1,2} \int_{D} d \vec{r}\left[\operatorname{Re} \delta \psi_{i^{\prime}} \frac{\delta H}{\delta \operatorname{Re} \psi_{i^{\prime}}}+\operatorname{Im} \delta \psi_{i^{\prime}} \frac{\delta H}{\delta \operatorname{Im} \psi_{i^{\prime}}}\right] \cong \int_{D} d \vec{r}\left[\operatorname{Re} \delta \psi_{i} \frac{\delta H}{\delta \operatorname{Re} \psi_{i}}+\operatorname{Im} \delta \psi_{i} \frac{\delta H}{\delta \operatorname{Im} \psi_{i}}\right] \\
& =\frac{1}{2} \int_{D} d \vec{r}\left[\delta \psi_{i} \frac{\delta H}{\delta \psi_{i}}+\delta \psi_{i}^{*} \frac{\delta H}{\delta \psi_{i}^{*}}\right]=-\frac{1}{2} \sum_{\alpha=x, y} \int_{D} d \vec{r} \sum_{j=1}^{N_{D}}\left[\gamma_{i \alpha}(\vec{r}, j)\left(L_{i}^{-1}\right)^{*} \sum_{\beta=x, y} \sum_{j^{\prime}=1}^{N_{D}} \gamma_{i \beta}^{*}\left(\vec{r}, j^{\prime}\right) v_{\beta}\left(j^{\prime}\right)+\text { c.c. }\right] \delta \eta_{\alpha}(j) \\
& \cong-\frac{1}{2} \sum_{\alpha=x, y} \int_{D} d \vec{r} \sum_{j=1}^{N_{D}}\left[\gamma_{i \alpha}(\vec{r}, j)\left(L_{i}^{-1}\right)^{*} \sum_{\beta=x, y} \gamma_{i \beta}^{*}(\vec{r}, j) v_{\beta}(j)+\text { c.c. }\right] \delta \eta_{\alpha}(j)=\sum_{\alpha=x, y} \sum_{j=1}^{N_{D}} \delta \eta_{\alpha}(j) \frac{\delta H}{\delta \eta_{\alpha}(j)} .
\end{aligned}
$$


Here we have defined the velocity of the $j$ th vortex as $v_{\alpha}(j)$. In the forth line, we have used the relation

$$
\partial_{t} \psi_{i}=\sum_{\alpha=x, y} \sum_{j=1}^{N_{D}} \gamma_{i \alpha}(\vec{r}, j) v_{\alpha}(j),
$$

which follows from Eq. (11), assuming that we can describe dynamics of the system in terms of defect motion. In the fifth line, we have ignored the contributions to $\delta H$ with $j \neq j^{\prime}$ by assuming that the contributions from the overlap between $\gamma_{i \alpha}(\vec{r}, j)$ and $\gamma_{i \beta}^{*}\left(\vec{r}, j^{\prime}\right)$ is very small for $j \neq j^{\prime}$ because the influence on the phase $\phi$ due to the presence of the $j$ th vortex is screened far away from the vortex $\left(\left|\vec{r}-\vec{r}_{j}\right| \gg R_{\mathrm{dis}}\right)$ by the other topological defects, where we have defined the characteristic distance among the vortices as $R_{\text {dis }}$. In the last line we have assumed that the free-energy functional $H$ is well described in terms of the configuration of the topological defects.

Because the displacement of the $j$ th vortex $\delta \eta_{\alpha}(j)$ is arbitrary, we find the following relation between the thermodynamic force on the $j$ th vortex $\frac{\delta H}{\delta \eta_{\alpha}(j)}$ and the velocity of the $j$ th vortex $v_{\beta}(j)$.

$$
\begin{aligned}
\frac{\delta H}{\delta \eta_{\alpha}(j)}= & -\frac{1}{2} \sum_{\beta=x, y}\left(\int _ { D } d \vec { r } \left[\gamma_{i \alpha}(\vec{r}, j)\right.\right. \\
& \left.\left.\times\left(L_{i}^{-1}\right)^{*} \gamma_{i \beta}^{*}(\vec{r}, j)+\text { c.c. }\right]\right) v_{\beta}(j) .
\end{aligned}
$$

Here the Goldstone mode due to the displacement of the $j$ th vortex is written as

$$
\gamma_{i \alpha}(\vec{r}, j)=-\partial_{\alpha} \psi_{i, j}(\vec{r}),
$$

in terms of the vortex solution $\psi_{i, j}(\vec{r})$ given in Eq. (17) below, where we have assumed that the correction of the vortex solution due to the other topological defects are small, which follows from $R_{\text {dis }} \gg \xi_{i} \cdot \psi_{i, j}(\vec{r})$ expresses the quasiequilibrium profile of $i$ th component complex order parameter around the $j$ th vortex located at $\vec{r}_{j}$.

We give the vortex solution $\psi_{i, j}(\vec{r})$ as

$$
\psi_{i, j}(\vec{r})=M_{i} \hat{F}_{0, i}(\rho) e^{i\left(n_{j} \phi+\theta_{j}\right)},
$$

where $\theta_{j}$ is constant and we have defined $\rho=\left|\vec{r}-\vec{r}_{j}\right|$ and $\phi=\tan ^{-1}\left(\frac{y-y_{j}}{x-x_{j}}\right)$. Here the real function $\hat{F}_{0, i j}(\rho)$ satisfies

$$
\left[\frac{K_{i}}{\left|a_{i}\right|}\left(\frac{1}{\rho} \frac{d}{d \rho} \rho \frac{d}{d \rho}-\frac{1}{\rho^{2}}\right)+\left(1-\hat{F}_{0, i}^{2}\right)\right] \hat{F}_{0, i}(\rho)=0 .
$$

$\hat{F}_{0, i}(\rho)$ behaves as $\hat{F}_{0, i}(\rho) \sim \rho$ for $\rho \ll \xi_{i}$ and $\hat{F}_{0, i}(\rho) \cong 1-$ $\frac{\xi_{i}^{2}}{2 \rho^{2}}$ for $\rho \gg \xi_{i}$.

With Eq. (16), we can estimate the following friction coefficient as

$$
\begin{aligned}
\int_{D} d \vec{r} & {\left[\gamma_{i \alpha}(\vec{r}, j)\left(L_{i}^{-1}\right)^{*} \gamma_{i \beta}^{*}(\vec{r}, j)+\text { c.c. }\right] } \\
= & \int_{D} d \vec{r}\left[2 \operatorname{Re} L_{i} \operatorname{Re}\left(\nabla_{\alpha} \psi_{i, j} \nabla_{\beta} \psi_{i, j}^{*}\right)\right. \\
& \left.-2 \operatorname{Im} L_{i} \operatorname{Im}\left(\nabla_{\alpha} \psi_{i, j} \nabla_{\beta} \psi_{i, j}^{*}\right)\right] /\left|L_{i}\right|^{2}
\end{aligned}
$$

$$
\begin{aligned}
& =\int_{D} d \vec{r} M_{i}^{2}\left(\operatorname { R e } L _ { i } \left\{\left[\frac{d}{d \rho} \hat{F}_{0, i}(\rho)\right]^{2}\right.\right. \\
& \left.+\left[\frac{1}{\rho} \hat{F}_{0, i}(\rho)\right]^{2}\right\}\left(\begin{array}{ll}
1 & 0 \\
0 & 1
\end{array}\right)_{\alpha \beta}+2 n_{j} \frac{1}{\rho} \operatorname{Im} L_{i} \hat{F}_{0, i}(\rho) \\
& \left.\quad \times \frac{d}{d \rho} \hat{F}_{0, i}(\rho)\left(\begin{array}{cc}
0 & 1 \\
-1 & 0
\end{array}\right)_{\alpha \beta}\right)\left(\frac{1}{\left|L_{i}\right|^{2}}\right) \\
& =\left(\frac{2 \pi M_{i}^{2}}{\left|L_{i}\right|^{2}}\right)\left(\begin{array}{cc}
\operatorname{Re} L_{i} C_{i} & n_{j} \operatorname{Im} L_{i} \\
-n_{j} \operatorname{Im} L_{i} & \operatorname{Re} L_{i} C_{i}
\end{array}\right)_{\alpha \beta}
\end{aligned}
$$

where we have introduced the coefficients $C_{i}$ as

$$
\begin{aligned}
C_{i} & =\int_{0}^{R_{\max }} d \rho \rho\left\{\left[\frac{d}{d \rho} \hat{F}_{0, i}(\rho)\right]^{2}+\left[\frac{1}{\rho} \hat{F}_{0, i}(\rho)\right]^{2}\right\} \\
& \cong \ln \left(R_{\max } / \xi_{i}\right) .
\end{aligned}
$$

Here we set the upper limit of the integral to the cutoff length $R_{\max }$. We can estimate $R_{\max }$ as follows. If there is a single vortex in the $i$-th component domain, $R_{\max }$ is of the order of the domain size. If there are many vortices in the domain, $R_{\max }$ is of the order of $R_{\text {dis }}$ due to the assumption that the influence on the phase $\phi$ due to the presence of the $j$ th vortex is screened far away from the vortex $\left(\rho \gg R_{\text {dis }}\right)$, which we have already mentioned below Eq. (13). We have rewritten the integral in polar coordinates and integrated in $\theta$ in deriving the forth line of Eq. (19). The following formulas have been used to evaluate each component for $\alpha=x, y$ and $\beta=x, y$ in deriving the third line of Eq. (19),

$$
\begin{aligned}
& \operatorname{Im}\left(\nabla_{x} \psi_{i, j} \nabla_{y} \psi_{i, j}^{*}\right) \\
& =M_{i}^{2}\left[\cos \phi \frac{d}{d \rho} \hat{F}_{0, i}(\rho)-i n_{j} \frac{\sin \phi}{\rho} \hat{F}_{0, i}(\rho)\right] \\
& \times\left[\sin \phi \frac{d}{d \rho} \hat{F}_{0, i}(\rho)-i n_{j} \frac{\cos \phi}{\rho} \hat{F}_{0, i}(\rho)\right] \\
& =-i n_{j} M_{i}^{2} \frac{1}{\rho} \hat{F}_{0, i}(\rho) \frac{d}{d \rho} \hat{F}_{0, i}(\rho), \\
& \operatorname{Re}\left(\nabla_{x} \psi_{i, j} \nabla_{x} \psi_{i, j}^{*}+\nabla_{y} \psi_{i, j} \nabla_{y} \psi_{i, j}^{*}\right) \\
& =M_{i}^{2}\left\{\left[\cos \phi \frac{d}{d \rho} \hat{F}_{0, i}(\rho)-i n_{j} \frac{\sin \phi}{\rho} \hat{F}_{0, i}(\rho)\right]\right. \\
& \times\left[\cos \phi \frac{d}{d \rho} \hat{F}_{0, i}(\rho)+i n_{j} \frac{\sin \phi}{\rho} \hat{F}_{0, i}(\rho)\right] \\
& +\left[\sin \phi \frac{d}{d \rho} \hat{F}_{0, i}(\rho)+i n_{j} \frac{\cos \phi}{\rho} \hat{F}_{0, i}(\rho)\right] \\
& \left.\times\left[\sin \phi \frac{d}{d \rho} \hat{F}_{0, i}(\rho)-i n_{j} \frac{\cos \phi}{\rho} \hat{F}_{0, i}(\rho)\right]\right\} \\
& =M_{i}^{2}\left\{\left[\frac{d}{d \rho} \hat{F}_{0, i}(\rho)\right]^{2}+\left[\frac{1}{\rho} \hat{F}_{0, i}(\rho)\right]^{2}\right\}, \\
& \int_{D} d \vec{r}\left[\operatorname{Re} L_{i} \operatorname{Re}\left(\nabla_{x} \psi_{i, j} \nabla_{x} \psi_{i, j}^{*}\right)\right] /\left|L_{i}\right|^{2} \\
& =\int_{D} d \vec{r}\left[\operatorname{Re} L_{i} \operatorname{Re}\left(\nabla_{y} \psi_{i, j} \nabla_{y} \psi_{i, j}^{*}\right)\right] /\left|L_{i}\right|^{2} .
\end{aligned}
$$


Using Eq. (15) and Eq. (19), we obtain the $\alpha$ th component of the velocity of the $j$ th vortex in the $i$ th component domain $D$ as

$$
\begin{aligned}
v_{\alpha}(j)= & -\frac{\left|L_{i}\right|^{2}}{\pi M_{i}^{2}} \frac{1}{\left(\operatorname{Re} L_{i} C_{i}\right)^{2}+\left(\operatorname{Im} L_{i}\right)^{2}} \\
& \times \sum_{\beta=x, y}\left(\begin{array}{cc}
\operatorname{Re} L_{i} C_{i} & -n_{j} \operatorname{Im} L_{i} \\
n_{j} \operatorname{Im} L_{i} & \operatorname{Re} L_{i} C_{i}
\end{array}\right)_{\alpha \beta} \frac{\delta H}{\delta \eta_{\beta}(j)} .
\end{aligned}
$$

Next we derive the equation of motion for an interface between a first-component domain and a second-component domain. In two-dimensional space, the interface is a line defect and parametrized as

$$
\vec{r}(l)=(x(l), y(l)),
$$

using the parameter $l$, which satisfies

$$
\left|\frac{d \vec{r}}{d l}\right|=1 .
$$

The deviation of $\psi_{i}(i=1,2)$ due to the displacement of the interface is given by

$$
\delta \psi_{i}(\vec{r})=\int d l \gamma_{i}(\vec{r}, l) \delta \eta(l),
$$

where we have introduced $\delta \eta(l)$ to denote the normal component of the interface deviation as

$$
\delta \eta(l)=\delta \vec{x}(l) \cdot \vec{n},
$$

where $\delta \vec{x}(l)$ is the displacement vector of the interface at the point $l$ and $\vec{n}$ is the unit vector, which is normal to the interface and directed toward the second component domain. Here we have defined the Goldstone mode due to the interface deviation as

$$
\begin{aligned}
& \gamma_{1}(\vec{r}, l)=-e^{i \theta_{1}(\vec{r}(l))} \frac{d}{d w} \psi_{1}(w(\vec{r})) \delta(l-l(\vec{r})), \\
& \gamma_{2}(\vec{r}, l)=-e^{i \theta_{2}(\vec{r}(l))} \frac{d}{d w} \psi_{2}(w(\vec{r})) \delta(l-l(\vec{r})) .
\end{aligned}
$$

Here $\theta_{i}(\vec{r}(l))$ is the phase of the complex field around the point $\vec{r}(l)$, and $\psi_{i}(w(\vec{r}))$ is the interface solution in the $i$ th component domain determined by Eqs. (B1)-(B3) in the Appendix. And we have introduced the coordinates $w(\vec{r})$ along the normal to the interface and $l(\vec{r})$ parallel to the interface so that $w(\vec{r})$ and $l(\vec{r})$ constitute a local orthonormal coordinate system near the interface and the region $w>0$ $(w<0)$ is occupied by the second (first) component domain, where the boundary conditions given by Eqs. (B2) and (B3) are appropriate. In this case, we assume that the $i$ th component of interface profile is well described by the 1D profile $e^{i \theta_{i}(\vec{r}(l))} \psi_{i}(w(\vec{r}))$ near the interface. Then by noting the variable $w$ decreases by $\varepsilon$ when we deviate the position of the interface by $\varepsilon$ to the positive direction (toward the second-component domain) at the interface point $l$, we obtain the expression given by Eqs. (28) and (29). The perturbation on the $1 \mathrm{D}$ profile due to the variation of the phase field or the curvature around the interface can be neglected in the lowest order of $\xi_{i}$ because the characteristic scale of these perturbations are very large compared to $\xi_{i}$.

Employing the same method by which we have derived Eq. (13), we can obtain the relation for the interface velocity as

$$
-\frac{1}{2} \int d \vec{r} \sum_{i^{\prime}=1,2}\left[\sum_{\alpha=x, y} \int d l \gamma_{i^{\prime} \alpha}(\vec{r}, l) \delta \eta_{\alpha}(l)\left(L_{i^{\prime}}^{-1}\right)^{*} \sum_{\beta=x, y} \int d l^{\prime} \gamma_{i^{\prime} \beta}^{*}\left(\vec{r}, l^{\prime}\right) v_{\beta}\left(l^{\prime}\right)+\text { c.c. }\right]=\sum_{\alpha=x, y} \int d l \delta \eta_{\alpha}(l) \frac{\delta H}{\delta \eta_{\alpha}(l)} .
$$

From this equation, we obtain the interface velocity along the unit vector $\vec{n}(l)$ as

$$
v(l)=-\left(\sum_{i^{\prime}=1,2} \frac{\operatorname{Re} L_{i^{\prime}}}{K_{i^{\prime}}\left|L_{i}\right|^{2}} \kappa_{i^{\prime}}\right)^{-1} \frac{\delta H}{\delta \eta(l)} .
$$

Here we have used the following relation:

$$
\begin{aligned}
\int d \vec{r} \sum_{i^{\prime}=1,2}\left[\gamma_{i^{\prime}}(\vec{r}, l)\left(L_{i^{\prime}}^{-1}\right)^{*} \gamma_{i^{\prime}}^{*}\left(\vec{r}, l^{\prime}\right)+\text { c.c. }\right] & =\int d \vec{r} \sum_{i^{\prime}=1,2}\left[\left(2 \operatorname{Re} L_{i^{\prime}}\left(\frac{d}{d w} \psi_{i^{\prime}}(w(\vec{r}))\right)^{2} \delta(l-l(\vec{r})) \delta\left(l^{\prime}-l(\vec{r})\right)\right) /\left|L_{i^{\prime}}\right|^{2}\right] . \\
& =\delta\left(l-l^{\prime}\right) \sum_{i^{\prime}=1,2}\left(\frac{2 \operatorname{Re} L_{i^{\prime}}}{K_{i^{\prime}}\left|L_{i^{\prime}}\right|^{2}} \kappa_{i^{\prime}}\right),
\end{aligned}
$$

where the coefficients $\kappa_{i}$ for $i=1,2$ are given as

$$
\kappa_{i}=\int d w K_{i}\left(\frac{d}{d w} \psi_{i}\right)^{2} .
$$

\section{DETERMINATION OF THE STATIC INTERACTIONS BETWEEN TOPOLOGICAL DEFECTS}

In this section, we will derive the static interaction between the topological defects. For simplicity, we consider a case where the whole system is divided into a first-component domain $D_{1}$ and a second-component domain $D_{2}$. It is easy to extend the result of this section to the cases with arbitrary number of domains.

In the thin-interface limit $R_{\max } \gg \xi$, the free energy is written in terms of the configuration of topological defects as

$$
\begin{aligned}
H= & \sum_{i=1,2}\left[\frac{1}{2} K_{i} M_{i}^{2} \int_{D_{i}} d \vec{r}\left|\vec{\nabla} \phi_{i}\right|^{2}\right]+\int d l \sigma \\
& +E_{1} V_{1}+E_{2} V_{2}+N_{1} E_{1 c}+N_{2} E_{2 c} .
\end{aligned}
$$


We have introduced the phase variable $\phi_{i}$ in the domain $D_{i}$ except near the interface and the vortices as

$$
\psi_{i}=M_{i} e^{i \phi_{i}} .
$$

The first term in the right-hand side is not well defined because $\phi_{i}$ is not defined near the core region of the radius $\xi_{i}$ around a quantized vortex. We will define this quantity more precisely in terms of the field $\chi$ later in Eqs. (39) and (40). Here $\sigma=\kappa_{1}+\kappa_{2}$ is the interface tension between two domains, which is derived in Appendix B. $E_{i}=\frac{a_{i}}{2} M_{i}^{2}+\frac{g_{i, i}}{4} M_{i}^{4}$ is the free-energy density for uniform $i$ th-component domain, $V_{i}$ is the total volume of the $i$ th-component domain, and $E_{i c}$ is the core energy of a vortex in the $i$ th-component domain. The coordinate $l$ parametrizes the interface between the domains $D_{1}$ and $D_{2}$, as defined in Eqs. (25) and (26).

Here, in the limit $R_{\max } \gg \xi$, we expect the situation that the correction to the interface and vortex profile due to the interaction among the topological defects is very small and the order parameters satisfy $\left(\left|\psi_{1}\right|,\left|\psi_{2}\right|\right)=\left(M_{1}, 0\right)$ or $\left(\left|\psi_{1}\right|,\left|\psi_{2}\right|\right)=\left(0, M_{2}\right)$ except near the topological defects, as mentioned in the previous sections. We note that there also exist short-ranged interactions between defects due to the amplitude modulation of the order parameter far from defects, which decay as $\exp [-r / \xi]$, where $r$ is the distance between the points where the defects are located $[1,7]$. We have ignored these short-ranged interactions because of the above assumption $R_{\max } \gg \xi$. We will see that the difference between $E_{1}$ and $E_{2}$ drives interface motion as in Eq. (53) below. Therefore, we require that $E_{1}-E_{2}$ is not large so that the the assumption that the timescale of interface motion is much larger than the decay times of other excitations.

First we will construct the free-energy minimum configuration of the field $\phi_{i}$ in the presence of the topological defects. We require the boundary condition

$$
\vec{n} \cdot \vec{\nabla} \phi_{i}=0
$$

at the interface. We will see that the configuration $\phi_{i}$ by the following equation minimizes the free energy.

$$
\vec{\nabla} \phi_{i}=\vec{\nabla} \times\left(\vec{e}_{z} \chi_{i}\right)
$$

where we have introduced the scalar field $\chi_{i}$, which is defined inside the $i$ th domain and satisfies

$$
\begin{aligned}
& \partial_{x} \chi_{i}=-\partial_{y} \phi_{i}, \quad \partial_{y} \chi_{i}=\partial_{x} \phi_{i}, \\
& -\nabla^{2} \chi_{i}=2 \pi \sum_{j} n_{j} S\left(\vec{r}-\vec{r}_{j}\right),
\end{aligned}
$$

to describe the vorticity due to the presence of the quantized vortices. Here we have defined $S\left(\vec{r}-\vec{r}_{j}\right)$ as

$$
S\left(\vec{r}-\vec{r}_{j}\right)=\left\{\begin{array}{ll}
1 /\left(\pi \xi_{i}^{2}\right) & \left|\vec{r}-\vec{r}_{j}\right| \leqslant \xi_{i} \\
0 & \text { otherwise }
\end{array},\right.
$$

in order to express the rotation of the phase field a quantized vortex outside the core regions. Equation (40) corresponds to the Poisson's equation for charges with finite sizes. We note that $\chi_{i}$ is well-defined in the domain $D_{i}$ except near the interface if $\xi_{i}$ is finite. We will take the limit $\xi_{i} \rightarrow 0$ for the vortex core radius after calculating the force on a vortex as in Eq. (59).
Here we have introduced $\vec{e}_{z}$, which is perpendicular to the $x y$ plane, to simplify the notation and $\vec{\nabla} \times\left(\vec{e}_{z} \chi_{i}\right)$ is the external product between the vectors $\vec{\nabla}$ and $\vec{e}_{z} \chi_{i}$ in three-dimensional space. To satisfy Eq. (37), the boundary condition for $\chi_{i}$ is given by

$$
\chi_{i}=\text { const, }
$$

which is equivalent to

$$
\vec{\nabla} \chi_{i} \| \vec{n}
$$

on the interface. Here we can set

$$
\chi_{i}=0
$$

at the interface without loss of generality.

Now we can prove that $\phi_{i}$ determined by Eqs. (37), (39), and (40) minimizes the free energy as follows. The variation of the gradient energy due to the variation of the phase variable is given as

$$
\begin{aligned}
& \delta\left[\int_{D_{i}} d \vec{r}\left|\vec{\nabla} \phi_{i}\right|^{2}\right] \\
& \quad=-2 \int_{D_{i}} d \vec{r}\left[\nabla^{2} \phi_{i}\right] \delta \phi_{i}+2 \int d l\left[\vec{n} \cdot \vec{\nabla} \phi_{i}\right] \delta \phi_{i} .
\end{aligned}
$$

Here $\vec{n}$ is the unit vector, which is perpendicular to the interface and is directed toward the outside of the domain $D_{i}$. From the above equation, we can see that it is needed to require $\vec{n} \cdot \vec{\nabla} \phi_{i}=0$ so that the gradient energy is minimized. In deriving Eq. (45), we have used the following identity:

$$
\begin{aligned}
& \int d l\left[\vec{n} \cdot \vec{\nabla} \phi_{i}\right] \delta \phi_{i} \\
& \quad=\int_{D_{i}} d \vec{r} \delta \phi_{i} \nabla^{2} \phi_{i}+\int_{D_{i}} d \vec{r}\left(\vec{\nabla} \delta \phi_{i}\right) \cdot\left(\vec{\nabla} \phi_{i}\right) .
\end{aligned}
$$

From Eq. (39), we obtain

$$
\int_{D_{i}} d \vec{r}\left|\vec{\nabla} \phi_{i}\right|^{2}=\int_{D_{i}} d \vec{r}\left|\vec{\nabla} \chi_{i}\right|^{2}
$$

and

$$
\nabla^{2} \phi_{i}=-\left(\partial_{x} \partial_{y}-\partial_{y} \partial_{x}\right) \chi_{i}=0,
$$

except near the quantized vortices. From Eqs. (37), (45), and (48), we conclude that the free-energy functional is minimized with respect to the variation of phase variables except near the vortices.

When we calculate the defect velocities later, we will assume that the phase $\phi_{i}$ is determined by Eq. (39), and Eq. (40) under the boundary condition given by Eq. (37) because the field $\phi_{i}$ relaxes to its minimum much faster than defect motion. We also note that we can take the variations of $\phi_{i}$ for $(i=1,2)$ independently because the first component and the second component are completely phase-separated.

We note that Eq. (48) for $\chi_{i}$ for each component under the boundary condition given by Eq. (44) is analogous to the Poisson's equation for electric field in a vacuum domain $D_{i}$ surrounded by a metallic object. In this analogy, the $j$ th electric charge is located at $\vec{r}_{j}$ and Eq. (47) corresponds to electrostatic 
energy. Unfortunately we cannot solve Eq. (48) analytically for general configurations of interfaces as it is the case in electrostatics. We will treat several cases where we can solve Eq. (48) analytically in Sec. V.

Now we can calculate the free-energy deviation due to the deformation of the domain $D_{i}$, which is required to obtain the interface velocity as in Eq. (32). Here we fix the positions of the vortices. We denote the deformed domain as $D_{i}^{\prime}$. Due to the change of the boundary condition on the domain $D_{i}$, the field $\chi$ also changes to $\chi^{\prime}$ in the first order of the magnitude of the deviation. Now we can prove the following relation

$$
\begin{aligned}
& \int_{D_{i}^{\prime}} d \vec{r}\left|\nabla \chi_{i}^{\prime}\right|^{2}-\int_{D_{i}} d \vec{r}\left|\nabla \chi_{i}\right|^{2} \\
& = \pm \int_{\partial D_{i}} d l \delta \eta(l)\left|\nabla \chi_{i}\right|^{2}+O\left(\delta \eta^{2}\right),
\end{aligned}
$$

where we take the sign + for $i=1$ and the sign - for $i=2$. The normal component of the interface deviation $\delta \eta(l)$ has been defined in Eq. (28). The proof of Eq. (49) is shown below.

$$
\begin{aligned}
\int_{D_{i}^{\prime}} d \vec{r}\left|\nabla \chi_{i}^{\prime}\right|^{2}-\int_{D_{i}} d \vec{r}\left|\nabla \chi_{i}\right|^{2} & = \pm \int_{\partial D_{i}} d l \delta \eta(l)\left|\nabla \chi_{i}\right|^{2}+\int_{D_{i} \cap D_{i}^{\prime}} d \vec{r}\left(\left|\nabla \chi_{i}^{\prime}\right|^{2}-\left|\nabla \chi_{i}\right|^{2}\right)+O\left(\delta \eta^{2}\right) \\
& = \pm \int_{\partial D_{i}} d l \delta \eta(l)\left|\nabla \chi_{i}\right|^{2}+2 \int_{D_{i} \cap D_{i}^{\prime}} d \vec{r}\left(-\chi_{i} \nabla^{2} \delta \chi_{i}\right)+O\left(\delta \eta^{2}\right) \\
& = \pm \int_{\partial D_{i}} d l \delta \eta(l)\left|\nabla \chi_{i}\right|^{2}+O\left(\delta \eta^{2}\right)
\end{aligned}
$$

Here $\partial D_{i}$ is the boundary between the domains and $\delta \chi_{i}=$ $\chi_{i}^{\prime}-\chi_{i}$. In the second line, the first term expresses the deviation due to the changing of the integration domain and the second term is the deviation due to the changing of $\chi$ inside the domain $D_{i} \cap D_{i}^{\prime}$. In the third line we have performed integration by part by employing $\chi_{i} \vec{\nabla} \delta \chi_{i}=0+O\left(\delta \eta^{2}\right)$ on the boundary of $D_{i} \cap D_{i}^{\prime}$ because $\chi_{i}=O(\delta \eta)$ on the boundary of $D_{i} \cap D_{i}^{\prime}$, which can be shown from the boundary condition given by Eq. (44). In the fourth line we have used the equation

$$
\nabla^{2} \delta \chi_{i}=0
$$

which means that the vortices are fixed in the process of the virtual displacement of the interface.

Then, using Eq. (49), we obtain

$$
\frac{\delta H}{\delta \eta(l)}=\frac{K_{1} M_{1}^{2}}{2}\left|\nabla \chi_{1}\right|^{2}-\frac{K_{2} M_{2}^{2}}{2}\left|\nabla \chi_{2}\right|^{2}+\sigma K+E_{1}-E_{2} .
$$

Here the curvature $K$ of the interface is defined as $K=\vec{\nabla} \cdot \vec{n}$. The first two terms in this equation indicate that the phase gradient in the $i$ th-component domain along the interface exerts the negative normal thermodynamic force which tends to attract the interface toward the inside of the $i$ th-component domain. The term $\sigma K$ indicates that the interface evolves so that the length of the interface decreases. The last two terms indicate that the difference between the free-energy densities of the two component drives interface motion.

Using Eqs. (32) and (52), we obtain the interface velocity as

$$
\begin{aligned}
v(l)= & -\left(\sum_{i=1}^{2} \frac{\operatorname{Re} L_{i}}{K_{i}\left|L_{i}\right|^{2}} \kappa_{i}\right)^{-1}\left[\frac{K_{1} M_{1}^{2}}{2}\left|\nabla \chi_{1}\right|^{2}\right. \\
& \left.-\frac{K_{2} M_{2}^{2}}{2}\left|\nabla \chi_{2}\right|^{2}+\sigma K+E_{1}-E_{2}\right] .
\end{aligned}
$$

Here we recall that the fields $\chi_{1}$ and $\chi_{2}$ are determined by Eq. (40) in terms of the positions of the vortices under the boundary condition given by Eq. (44) at the interface. Therefore, the term $-\left(\sum_{i=1}^{2} \frac{\operatorname{Re} L_{i}}{K_{i}\left|L_{i}\right|^{2}} \kappa_{i}\right)^{-1}$ $\left(\frac{K_{1} M_{1}^{2}}{2}\left|\nabla \chi_{1}\right|^{2}-\frac{K_{2} M_{2}^{2}}{2}\left|\nabla \chi_{2}\right|^{2}\right)$ describes the interface velocity due to the presence of the vortices. Qualitatively, this term expresses attractive interactions between the domain boundary and vortices inside the domain, which are analogous to an attractive interaction between an electric charge and a metallic object due to Maxwell's stress.

Next we calculate the velocity of the $j$ th vortex given by Eq. (24). In order to calculate $\delta H / \delta \eta_{\beta}(j)$, we express the gradient term in the free energy as

$$
\begin{aligned}
& \frac{1}{2} K_{i} M_{i}^{2} \int_{D_{i}} d \vec{r}\left|\nabla \chi_{i}\right|^{2} \\
& \quad=-\frac{1}{2} K_{i} M_{i}^{2} \int_{D_{i}} d \vec{r} \chi_{i} \nabla^{2} \chi_{i} \\
& \quad=\pi K_{i} M_{i}^{2} \sum_{j=1}^{N_{i}} \int_{\left|\vec{r}-\vec{r}_{j}\right| \leqslant \xi_{i}} d \vec{r} \frac{1}{\pi \xi_{i}^{2}} n_{j} \chi_{i}(\vec{r}) \\
& \cong \pi K_{i} M_{i}^{2} \sum_{j=1}^{N_{i}} n_{j} \chi_{i}\left(\vec{r}_{j}\right),
\end{aligned}
$$

where we have performed partial integration using Eq. (44), and used Eq. (40). In the forth line, we have taken the thininterface limit $\xi_{i} \rightarrow 0$.

We note that the term $n_{j} \chi_{i}\left(\vec{r}_{j}\right)$ contains the constant self energy of the charge $j$, which diverges as $\ln \left(C / \xi_{i}\right)$ in the thin-interface limit, where $C$ is a constant which has dimension of length. However, this constant divergence in the gradient energy does not affect the calculation of the force on a vortex or an interface because the force is well-defined in the limit $\xi_{i} \rightarrow$ 0 , as one can verify it in the process deriving Eqs. (64), (67), and (75). Thus, we will neglect this constant divergence in the limit $\xi_{i} \rightarrow 0$. The similar situation occurs for electric energy of a point charge in electrostatic. Though the electric energy contains constant divergence due to the contribution of the self 
energy in the limit that the radius of the point charge goes to zero, the force on the charge is well-defined in the limit.

Here, by noting that Eq. (40) is linear, we can express the scalar field $\chi_{i}\left(\vec{r}_{j}\right)$ as

$$
\chi_{i}\left(\vec{r}_{j}\right)=\sum_{j^{\prime}=1}^{N_{i}} n_{j^{\prime}} \zeta_{i}\left(\vec{r}_{j}, \vec{r}_{j^{\prime}}\right),
$$

in terms of superposition of the solutions $\zeta_{i}\left(\vec{r}, \vec{r}_{j^{\prime}}\right)$ for a single vortex, which has charge 1 and is located at $\vec{r}_{j^{\prime}}$ in the $i$ th domain. $\zeta_{i}\left(\vec{r}, \vec{r}_{j^{\prime}}\right)$ is determined by

$$
-\nabla^{2} \zeta_{i}\left(\vec{r}, \vec{r}_{j^{\prime}}\right)=2 \pi S\left(\vec{r}-\vec{r}_{j^{\prime}}\right),
$$

where the Laplacian $\nabla^{2}$ operates on $\vec{r}$, under the boundary condition

$$
\zeta_{i}\left(\vec{r}, \vec{r}_{j^{\prime}}\right)=0
$$

where $\vec{r}$ is on the boundary of $i$ th domain. In electrostatics [14], it is known that $\zeta_{i}\left(\vec{r}_{j}, \vec{r}_{j^{\prime}}\right)$ satisfies the following reciprocal relation

$$
\zeta_{i}\left(\vec{r}_{j}, \vec{r}_{j^{\prime}}\right)=\zeta_{i}\left(\vec{r}_{j^{\prime}}, \vec{r}_{j}\right),
$$

which is called Green's reciprocity theorem. Thus, the freeenergy deviation due to the displacement of the $j$ th vortex in the $i$ th component domain is given by

$$
\frac{\delta H}{\delta \eta_{\beta}(j)}=2 \pi K_{i} M_{i}^{2} n_{j} \nabla_{\beta} \chi_{i}\left(\vec{r}_{j}\right) .
$$

Here we can see that $\frac{\delta H}{\delta \eta_{\beta}(j)}$ is determined by the positions of the interface and the other vortices because the phase $\chi_{i}$ is determined by Eqs. (39) and (40). This term expresses not only interactions between vortices but also an attractive interaction between the domain boundary and a vortex with any charge inside the domain, which is analogous to an attractive force on an electric charge towards a metallic boundary due to induced charge.

Finally, by substituting the above equation into Eq. (24), we obtain the vortex velocity of the $j$ th vortex in the $i$ th component domain as

$$
\begin{aligned}
\vec{v}_{j}= & -2\left|L_{i}\right|^{2} \frac{K_{i}}{\left(C_{i} \operatorname{Re} L_{i}\right)^{2}+\left(\operatorname{Im} L_{i}\right)^{2}} \\
& \times\left[C_{i} n_{j} \operatorname{Re} L_{i} \vec{\nabla} \chi_{i}\left(\vec{r}_{j}\right)+\operatorname{Im} L_{i} \vec{e}_{z} \times \vec{\nabla} \chi_{i}\left(\vec{r}_{j}\right)\right] \\
= & -2\left|L_{i}\right|^{2} \frac{K_{i}}{\left(C_{i} \operatorname{Re} L_{i}\right)^{2}+\left(\operatorname{Im} L_{i}\right)^{2}} \\
& \times\left[C_{i} n_{j} \operatorname{Re} L_{i} \vec{e}_{z} \times \vec{\nabla} \phi_{i}\left(\vec{r}_{j}\right)-\operatorname{Im} L_{i} \vec{\nabla} \phi_{i}\left(\vec{r}_{j}\right)\right] .
\end{aligned}
$$

Here the $z$ direction can be regarded as the direction of vorticities. In superfluids, $\vec{\nabla} \phi_{i}$ is proportional to the velocity of superflow in the $i$ th component. The first contribution that is proportional to $\vec{e}_{z} \times \vec{\nabla} \phi_{i}\left(\vec{r}_{j}\right)$ can be regarded as an analog of motion of a rotating object in uniform flow due to Magnus effect [15]. We note that the $\beta$ component of the first contribution is proportional to $\frac{\delta H}{\delta \eta_{\beta}(j)}$, which expresses interactions between vortices and attractive interactions between the domain boundary and vortices with any charge inside the domain as mentioned above. The second contribution is proportional to the velocity of superflow, and this indicates that a vortex is dragged by superflow.

\section{EXAMPLES}

Though we cannot solve Eq. (40) for the field $\chi$ under general boundary conditions, we give some examples which we can treat analytically.

\section{A. Vortex motion in a single domain}

Here we consider cases where the whole space is occupied by the first-component domain and there exist several vortices. We note that Eq. (60) for the vortex velocity under the phase gradient $\nabla_{\beta} \chi$ in the one-component complex GinzburgLandau equation was obtained by many authors [2,7]. We denote the position and the topological charge of these vortices as $\vec{r}_{i}$ and $n_{i}$. Then we can solve Eq. (40) to determine $\chi_{1}(\vec{r})$ as

$$
\chi_{1}(\vec{r})=-\sum_{i} n_{i} \ln \left(\frac{\left|\vec{r}-\vec{r}_{i}\right|}{r_{0}}\right) .
$$

When there are only two vortices with opposite signs (a vortex with sign +1 at $\vec{r}_{1}$ and a vortex with sign -1 at $\vec{r}_{2}$ ), the velocities of the two vortices are

$$
\begin{aligned}
\vec{v}_{1}= & 2\left|L_{i}\right|^{2} \frac{K_{i}}{\left(C_{i} \operatorname{Re} L_{i}\right)^{2}+\left(\operatorname{Im} L_{i}\right)^{2}} \\
& \times\left[-C_{i} \operatorname{Re} L_{i}\left(\frac{\vec{r}_{1}-\vec{r}_{2}}{\left|\vec{r}_{2}-\vec{r}_{1}\right|^{2}}\right)-\operatorname{Im} L_{i} \vec{e}_{z} \times\left(\frac{\vec{r}_{1}-\vec{r}_{2}}{\left|\vec{r}_{2}-\vec{r}_{1}\right|^{2}}\right)\right],
\end{aligned}
$$

$$
\begin{aligned}
\vec{v}_{2}= & 2\left|L_{i}\right|^{2} \frac{K_{i}}{\left(C_{i} \operatorname{Re} L_{i}\right)^{2}+\left(\operatorname{Im} L_{i}\right)^{2}} \\
& \times\left[-C_{i} \operatorname{Re} L_{i}\left(\frac{\vec{r}_{2}-\vec{r}_{1}}{\left|\vec{r}_{2}-\vec{r}_{1}\right|^{2}}\right)+\operatorname{Im} L_{i} \vec{e}_{z} \times\left(\frac{\vec{r}_{2}-\vec{r}_{1}}{\left|\vec{r}_{2}-\vec{r}_{1}\right|^{2}}\right)\right] .
\end{aligned}
$$

The distance between the two vortices evolves as

$$
\begin{aligned}
\frac{d}{d t}\left|\vec{r}_{1}-\vec{r}_{2}\right|= & -4\left|L_{i}\right|^{2} \frac{K_{i}}{\left(C_{i} \operatorname{Re} L_{i}\right)^{2}+\left(\operatorname{Im} L_{i}\right)^{2}} \\
& \times C_{i} \operatorname{Re} L_{i}\left(\frac{1}{\left|\vec{r}_{2}-\vec{r}_{1}\right|}\right) .
\end{aligned}
$$

We can see that the two vortices attract each other.

\section{B. Motion of a circular domain}

Here we consider the case that there is a circular domain of first-component domain and the outside of the domain is occupied by second component. We denote the center of this domain as $\overrightarrow{r_{0}}$. We assume that a vortex with charge $n_{\text {in }}$ is located at $\vec{r}_{0}$ and there exists superflow circulation $2 \pi n_{\text {out }}$ outside the circular domain. Then, the phase is given as

$$
\begin{aligned}
\phi_{\text {outside }}(\vec{r}) & =\sum_{i} n_{\text {out }} \theta(\vec{r}), \\
\phi_{\text {inside }}(\vec{r}) & =\sum_{i} n_{\text {in }} \theta(\vec{r}),
\end{aligned}
$$


where $\theta(\vec{r})$ is $\tan ^{-1}\left(\frac{y}{x}\right)$. The radius of the domain $R$ evolves as

$$
\begin{aligned}
\frac{d R}{d t}= & -\left(\sum_{i} \frac{2 \operatorname{Re} L_{i}}{K_{i}\left|L_{i}\right|^{2}} \kappa_{i}\right)^{-1}\left[\frac{K_{1} M_{1}^{2}}{2} \frac{n_{\mathrm{in}}^{2}}{R^{2}}\right. \\
& \left.-\frac{K_{2} M_{2}^{2}}{2} \frac{n_{\text {out }}^{2}}{R^{2}}+\frac{2}{R} \sigma+E_{1}-E_{2}\right] .
\end{aligned}
$$

Here, we can see the phase gradient along the interface, due to the vortex inside the circular domain or the superfluid circulation outside the domain, influences the motion of the interface. With the estimation $\sigma \propto K_{i} M_{i}^{2} / \xi$, we find that this effect becomes significant if $n_{\text {out }}$ or $n_{\text {in }}$ is on order of $\sqrt{R / \xi}$. However, we note that $\left|n_{\text {in }}\right| \sim \sqrt{R / \xi} \gg 1$ is somewhat an unrealistic situation because a vortex with $\left|n_{\text {in }}\right|>1$ is energetically unstable as is already noted in Sec. III. Madison et al. observed that a vortex lattice exists inside a BEC domain under rotation and there exists circulation of superflow [16]. $n_{\text {in }} \gg 1$ might correspond to cases with a vortex lattice inside a rotating domain with respect to the motion of the domain boundary.

\section{Initial velocities of a planar interface and vortices}

We consider a situation that a first-component domain in the region $y>0$ and a second-component domain in the region $y<0$ are separated by the interface $y=0$, and we assume that there are $N_{1}$ vortices in the first component domain and $N_{2}$ vortices in the second-component domain. In this section, we restrict ourselves to initial velocity of defects because, after the planar interface has deformed, we can no longer analytically solve Eq. (40) under the boundary condition given by Eq. (44). We denote the positions and the charges of the vortices as

$$
\vec{r}_{1, i}=\left(x_{1, i}, y_{1, i}\right), \quad n_{1, i}= \pm 1,
$$

for $i=1,2 \cdots, N_{1}$ in the first-component domain, and

$$
\vec{r}_{2, i}=\left(x_{2, i}, y_{2, i}\right), \quad n_{2, i}= \pm 1
$$

for $i=1,2, \cdots, N_{2}$ in the second-component domain. We can solve the scalar field $\chi_{1}$ and $\chi_{2}$ as

$$
\begin{aligned}
& \chi_{1}(\vec{r})=-\sum_{i=1}^{N_{1}} n_{1, i}\left[\ln \left(\frac{\left|\vec{r}-\vec{r}_{1, i}\right|}{r_{0}}\right)-\ln \left(\frac{\left|\vec{r}-\vec{s}_{1, i}\right|}{r_{0}}\right)\right] \quad(\text { for } y>0), \\
& \chi_{2}(\vec{r})=-\sum_{i=1}^{N_{2}} n_{2, i}\left[\ln \left(\frac{\left|\vec{r}-\vec{r}_{2, i}\right|}{r_{0}}\right)-\ln \left(\frac{\left|\vec{r}-\vec{s}_{2, i}\right|}{r_{0}}\right)\right] \quad(\text { for } y<0),
\end{aligned}
$$

under the boundary conditions

$$
\chi_{1}=0, \quad \chi_{2}=0 .
$$

at $y=0$. Here we have defined $\vec{s}_{1, i}$ and $\vec{s}_{2, i}$ as

$$
\begin{aligned}
& \vec{s}_{1, i}=\left(x_{1, i},-y_{1, i}\right), \\
& \vec{s}_{2, i}=\left(x_{2, i},-y_{2, i}\right) .
\end{aligned}
$$

We obtain the velocities of the $i$ th vortex in the first-component domain as

$$
\begin{aligned}
\vec{v}_{1, i}= & 2\left|L_{1}\right|^{2} \frac{K_{1}}{\left(C_{1} \operatorname{Re} L_{1}\right)^{2}+\left(\operatorname{Im} L_{1}\right)^{2}}\left(n_{1, i} C_{1} \operatorname{Re} L_{1}+\operatorname{Im} L_{1} \vec{e}_{z} \times\right) \\
& \times\left\{-n_{1, i} \frac{\left(\vec{r}_{1, i}-\vec{s}_{1, i}\right)}{\left|\vec{r}_{1, i}-\vec{s}_{1, i}\right|^{2}}+\sum_{j=1, j \neq i}^{N_{1}} n_{1, j}\left[\frac{\left(\vec{r}_{1, i}-\vec{r}_{1, j}\right)}{\left|\vec{r}_{1, i}-\vec{r}_{1, j}\right|^{2}}-\frac{\left(\vec{r}_{1, i}-\vec{s}_{1, j}\right)}{\left|\vec{r}_{1, i}-\vec{s}_{1, j}\right|^{2}}\right]\right\}
\end{aligned}
$$

We can also calculate the initial velocity of interface by substituting Eqs. (70) and (71) into Eq. (53). We mention that the vortex motion in a nondissipative Bose-Einstein condensation near a surface was treated by the matched asymptotic method [12]. They obtained the same phase field in the thin defect core limit, and it was shown that the vortex is driven to parallel to the surface, which qualitatively agrees with Eq. (75) after setting $\operatorname{Re} L_{1}=0$ formally. However, we note that our theory is not justified in the nondissipative case with $\operatorname{Re} L_{1}=0$.

\section{DISCUSSION AND CONCLUDING REMARKS}

In this paper, we have discussed dynamics of vortices and interfaces in the two-component complex Ginzburg-Landau model. We have derived the equations of motion of vortices and interfaces as Eqs. (32) and (60) under the scalar field $\chi_{i}$, which is related to the phase $\phi_{i}$ by Eq. (39) and determined by Eq. (40) under the boundary conditions given by Eq. (44). Vortex motion is driven by the phase gradient $\vec{\nabla} \chi_{i}$ around it, and interface motion is driven by the energy density difference $E_{1}-E_{2}$, the curvature $\sigma K$, and the phase gradients along it in the two domains. Equation (40) for $\chi_{i}$ under the boundary conditions given by Eq. (44) is analogous to the Poisson's equation for electric field in a vacuum domain $D_{i}$ surrounded by a metallic object. This analogy also indicates that vortices behave like electric charges in a vacuum domain surrounded by a metallic domain boundary and there is an attractive interaction between an interface and a quantized vortex with any charge. 
The two-component complex Ginzburg-Landau model with $\left|\operatorname{Re} L_{i}\right| \neq 0$ is used to describe the dissipative dynamics of two-component Bose-Einstein condensates. However, we should note that our theory depends on the assumption that, in the thin-interface limit, the time scale of motion defect motion is so long that it is well separated from time scales of the other excitation modes. Due to this assumption we have been able to ignore all the other excitations because they decay much faster than defect motion. In some numerical simulations of Bose-Einstein condensates, our assumption is not satisfied and interplays between Kelvin waves and vortex motion were observed [17]. We expect that our theory is justified for sufficiently slow defect motion in dissipative dynamics of two-component Bose-Einstein condensations, which is realized if the energy density difference $E_{1}-E_{2}$ is small and the distance between defects is large.

In this work, we have restricted ourselves to twodimensional space. In three-dimensional space, however, possible configurations of defects become more complicated, and dynamics would be richer. For example, a vortex line can penetrate an interface. Therefore, it would be interesting to extend this work to three-dimensional space.

\section{ACKNOWLEDGMENTS}

This study started with a conversation with Professor T. Ohta to whom the author is grateful for useful advices and guiding him to the field of defect dynamics. This work was supported by the JSPS Core-to-Core Program "International research network for non-equilibrium dynamics of soft matter." The author (S.Y.) was supported by the Japan Society for the Promotion of Science.

\section{APPENDIX A: ENERGY MINIMA OF THE FREE-ENERGY FUNCTIONAL}

The free-energy minima are taken at spatially uniform configurations $\psi_{i}(\vec{r})=\left(\psi_{1}^{\mathrm{min}}, \psi_{2}^{\mathrm{min}}\right)$ and the amplitudes $\left|\psi_{1}^{\mathrm{min}}\right|$ and $\left|\psi_{2}^{\mathrm{min}}\right|$ are determined by

$$
a_{1}\left|\psi_{1}^{\min }\right|+g_{1,1}\left|\psi_{1}^{\min }\right|^{3}+g_{1,2}\left|\psi_{1}^{\min }\right|\left|\psi_{2}^{\min }\right|^{2}=0,
$$

and

$$
a_{2}\left|\psi_{2}^{\min }\right|+g_{2,2}\left|\psi_{2}^{\min }\right|^{3}+g_{1,2}\left|\psi_{2}^{\min }\right|\left|\psi_{1}^{\min }\right|^{2}=0 .
$$

First we have the following single-component solutions given by

$$
\left(\left|\psi_{1}^{\min }\right|,\left|\psi_{2}^{\min }\right|\right)=\left(\sqrt{\frac{\left|a_{1}\right|}{g_{1,1}}}, 0\right),\left(0, \sqrt{\frac{\left|a_{2}\right|}{g_{2,2}}}\right) .
$$

The free energy densities for these single-component solutions are given by

$$
E_{1}^{\text {single }}=-\frac{a_{1}^{2}}{4 g_{1,1}}
$$

and

$$
E_{2}^{\text {single }}=-\frac{a_{2}^{2}}{4 g_{2,2}}
$$

respectively. Next we seek two-component solutions that satisfy both $\left|\psi_{1}^{\mathrm{min}}\right| \neq 0$ and $\left|\psi_{2}^{\mathrm{min}}\right| \neq 0$. Under this condition, the amplitudes are determined by

$$
\left(\begin{array}{ll}
g_{1,1} & g_{1,2} \\
g_{1,2} & g_{2,2}
\end{array}\right)\left(\begin{array}{l}
\left|\psi_{1}^{\min }\right|^{2} \\
\left|\psi_{2}^{\min }\right|^{2}
\end{array}\right)=-\left(\begin{array}{l}
a_{1} \\
a_{2}
\end{array}\right)
$$

We note that the two-component solutions determined by the above equation exist only if $g_{11} a_{2}-g_{12} a_{1} \geqslant 0$ and $g_{22} a_{1}-$ $g_{12} a_{2} \geqslant 0$. The free-energy density for these two-component solutions is given by

$$
E^{\text {multi }}=-\frac{1}{4}\left(\begin{array}{ll}
a_{1} & a_{2}
\end{array}\right)\left(\begin{array}{ll}
g_{1,1} & g_{1,2} \\
g_{1,2} & g_{2,2}
\end{array}\right)^{-1}\left(\begin{array}{l}
a_{1} \\
a_{2}
\end{array}\right) .
$$

Here we can show that $E^{\text {multi }}$ is larger than $E_{1}^{\text {single }}$ as

$$
\begin{aligned}
& E^{\text {multi }}-E_{1}^{\text {single }}=-\frac{1}{4\left(g_{1,1} g_{2,2}-g_{1,2}^{2}\right)}\left(\begin{array}{ll}
a_{1} & a_{2}
\end{array}\right) \\
& \times\left(\begin{array}{cc}
g_{2,2} & -g_{1,2} \\
-g_{1,2} & g_{1,1}
\end{array}\right)\left(\begin{array}{l}
a_{1} \\
a_{2}
\end{array}\right)+\frac{a_{1}^{2}}{4 g_{1,1}} \\
& =\frac{-g_{1,2}^{2} a_{1}^{2}+2 g_{1,1} g_{1,2} a_{1} a_{2}-g_{1,1}^{2} a_{2}^{2}}{4 g_{1,1}\left(g_{1,1} g_{2,2}-g_{1,2}^{2}\right)} \geqslant 0 \text {. }
\end{aligned}
$$

Here we have used the condition $-g_{1,1} g_{2,2}+g_{1,2}^{2} \geqslant 0$. In the same way, we can prove $E^{\text {multi }} \geqslant E_{2}^{\text {single }}$.

\section{APPENDIX B: INTERFACE TENSION IN THE TWO-COMPONENT GINZBURG-LANDAU EQUATION}

In this section we calculate the interface tension of an equilibrium solution where the two ordered phases coexist, which means that free-energy densities $E_{1}$ and $E_{2}$ of the two phases are equal. The small difference $E_{1}-E_{2}$ has been treated as a perturbative term on equilibrium solutions, which drives interface motion as in Eq. (32). We consider an equilibrium one-dimensional profile, which satisfies

$$
\begin{aligned}
& \left(\psi_{1}, \psi_{2}\right)=\left(M_{1}, 0\right) \quad(x \rightarrow-\infty), \\
& \left(\psi_{1}, \psi_{2}\right)=\left(0, M_{2}\right) \quad(x \rightarrow+\infty) .
\end{aligned}
$$

The profile can be determined by the free-energy minimum condition:

$$
\frac{\delta H}{\delta \psi_{i}^{*}}=a_{i} \psi_{i}+\sum_{j=1,2} g_{i, j} \psi_{i}\left|\psi_{j}\right|^{2}-K_{i} \frac{d^{2}}{d x^{2}} \psi_{i}=0
$$

for $i=1,2$. From this equation, we can show $\operatorname{Im} \psi_{i}=0$ for the equilibrium solution, which satisfies the boundary conditions given by Eqs. (B1) and (B2). The proof is as follows: From 
Eq. (B3), we obtain

$$
\begin{aligned}
0 & =\operatorname{Im} \int_{-\infty}^{x} d x \frac{\delta H}{\delta \psi_{1}^{*}} \psi_{1}^{*}=\operatorname{Im} \int_{-\infty}^{x} d x\left(a_{1} \psi_{1}+\sum_{j=1,2} g_{1, j} \psi_{1}\left|\psi_{j}\right|^{2}-K_{1} \frac{d^{2}}{d x^{2}} \psi_{1}\right) \psi_{1}^{*} \\
& =-K_{1} \operatorname{Im} \int_{-\infty}^{x} d x\left[\left(\frac{d^{2}}{d x^{2}} \psi_{1}\right) \psi_{1}^{*}+\left(\frac{d}{d x} \psi_{1}\right)\left(\frac{d}{d x} \psi_{1}^{*}\right)\right]=-K_{1} \operatorname{Im}\left[\psi_{1}^{*} \frac{d}{d x} \psi_{1}\right](x),
\end{aligned}
$$

where we have used the fact $-\operatorname{Im}\left[\psi_{1}^{*} \frac{d}{d x} \psi_{1}\right](x) \rightarrow 0$ in the limit $x \rightarrow-\infty$, which can be derived from the boundary condition given by Eq. (B2). This means $\frac{d \theta_{1}}{d x}=0$ and $\theta_{1}=$ const, where $\theta_{1}$ is the argument of the complex order parameter $\psi_{1}$. From Eq. (B1), $\theta_{1}$ must be 0 . Thus, we have proven $\operatorname{Im} \psi_{1}=0$. We can prove $\operatorname{Im} \psi_{2}=0$ in the same way.

Using Eq. (B3), we can derive the following relation:

$$
\begin{aligned}
0 & =\sum_{i=1,2} \int_{-\infty}^{x} d x\left(a_{i} \psi_{i}+\sum_{j=1,2} g_{i, j} \psi_{i}\left|\psi_{j}\right|^{2}-K_{i} \frac{d^{2}}{d x^{2}} \psi_{i}\right) \frac{d \psi_{i}}{d x} \\
& =\sum_{i=1,2}\left[\frac{1}{2} a_{i}\left|\psi_{i}\right|^{2}+\sum_{j=1,2} \frac{1}{4} g_{i, j}\left|\psi_{i}\right|^{2}\left|\psi_{j}\right|^{2}-\frac{1}{2} K_{i}\left(\frac{d}{d x} \psi_{i}\right)^{2}\right]-E_{1} .
\end{aligned}
$$

With this relation, we can calculate the interface tension in our system as

$\sigma=\int d x\left\{\sum_{i=1,2}\left[\frac{1}{2} a_{i}\left|\psi_{i}\right|^{2}+\sum_{j=1,2} \frac{1}{4} g_{i, j}\left|\psi_{i}\right|^{2}\left|\psi_{j}\right|^{2}+\frac{1}{2} K_{i}\left(\frac{d}{d x} \psi_{i}\right)^{2}\right]-E_{1}\right\}=\int d x \sum_{i=1,2} K_{i}\left(\frac{d}{d x} \psi_{i}\right)^{2}=\kappa_{1}+\kappa_{2}$.

Here $\kappa_{1}$ and $\kappa_{2}$ are defined in Eq. (34).

[1] K. Kawasaki and T. Ohta, Prog. Theor. Phys. 68, 129 (1982).

[2] A. Onuki, J. Low Temp. Phys. 51, 601 (1983).

[3] L. M. Pismen and J. Rubinstein, Physica D 47, 353 (1991).

[4] H. Pleiner, Phys. Rev. A 37, 3986 (1988).

[5] C. Denniston, Phys. Rev. B 54, 6272 (1996).

[6] G. Toth, C. Denniston, and J. M. Yeomans, Phys. Rev. Lett. 88, 105504 (2002).

[7] K. Kawasaki, Ann. Phys. 154, 319 (1984).

[8] K. Kawasaki and S. Iwamoto, Physica B+C 133, 76 (1985).

[9] J. I. Fukuda, Phys. Rev. E 59, 3275 (1999).

[10] T. Araki and H. Tanaka, Phys. Rev. Lett. 93, 015702 (2004).
[11] K. Sasaki, N. Suzuki, D. Akamatsu, and H. Saito, Phys. Rev. A 80, 063611 (2009).

[12] J. R. Anglin, Phys. Rev. A 65, 063611 (2002).

[13] C. J. Pethick and H. Smith, Bose-Einstein Condensation in Dilute Gases, 2nd ed. (Cambridge University Press, Cambridge, 2008).

[14] J. D. Jackson, Classical Electrodynamics, 3rd ed. (Wiley, Hoboken, 1998).

[15] L. D. Landau and E. M. Lifschitz, Fluid Mechanics, 2nd ed. (Butterworth-Heinemann, Oxford, 1987).

[16] K. W. Madison, F. Chevy, W. Wohlleben, and J. Dalibard, Phys. Rev. Lett. 84, 806 (2000).

[17] K. Kasamatsu, M. Machida, N. Sasa, and M. Tsubota, Phys. Rev. A 71, 043611 (2005). 